\title{
The HLA Class II Allele DRB1*1501 Is Over-Represented in Patients with Idiopathic Pulmonary Fibrosis
}

\author{
Jianmin Xue ${ }^{19}$, Bernadette R. Gochuico ${ }^{29}$, Ahmad Samer Alawad', Carol A. Feghali-Bostwick', Imre \\ Noth $^{3}$, Steven D. Nathan ${ }^{4}$, Glenn D. Rosen ${ }^{5}$, Ivan O. Rosas ${ }^{6}$, Sanja Dacic ${ }^{7}$, Iclal Ocak ${ }^{8}$, Carl R. Fuhrman ${ }^{8}$, \\ Karen T. Cuenco ${ }^{9}$, Mary A. Smith ${ }^{4}$, Susan S. Jacobs ${ }^{5}$, Adriana Zeevi ${ }^{7}$, Penelope A. Morel ${ }^{10}$, Joseph M. \\ Pilewski ${ }^{1}$, Vincent G. Valentine ${ }^{11}$, Kevin F. Gibson ${ }^{1}$, Naftali Kaminski ${ }^{1}$, Frank C. Sciurba ${ }^{1}$, Yingze Zhang ${ }^{1}$, \\ Steven R. Duncan ${ }^{1 *}$
}

1 Department of Medicine, University of Pittsburgh, Pittsburgh, Pennsylvania, United States of America, 2 Medical Genetics Branch, National Human Genome Research Institute, National Institutes of Health, Bethesda, Maryland, United States of America, 3 Department of Medicine, University of Chicago Medical Center, Chicago, Illinois, United States of America, 4 Advanced Lung Disease Program, Inova Fairfax Hospital, Falls Church, Virginia, United States of America, 5 Department of Medicine, Stanford University Medical Center, Stanford, California, United States of America, 6 Department of Medicine, Brigham and Women's Hospital, Harvard Medical School, Boston, Massachusetts, United States of America, 7 Department of Pathology, University of Pittsburgh, Pittsburgh, Pennsylvania, United States of America, 8 Department of Radiology, University of Pittsburgh, Pittsburgh, Pennsylvania, United States of America, 9 Department of Oral Biology-Dental Medicine and Human Genetics, University of Pittsburgh, Pittsburgh, Pennsylvania, United States of America, 10 Department of Immunology, University of Pittsburgh, Pittsburgh, Pennsylvania, United States of America, 11 Department of Medicine, University of Texas Medical Branch, Galveston, Texas, United States of America

\begin{abstract}
Background: Idiopathic pulmonary fibrosis (IPF) is a progressive and medically refractory lung disease with a grim prognosis. Although the etiology of IPF remains perplexing, abnormal adaptive immune responses are evident in many afflicted patients. We hypothesized that perturbations of human leukocyte antigen (HLA) allele frequencies, which are often seen among patients with immunologic diseases, may also be present in IPF patients.

Methods/Principal Findings: HLA alleles were determined in subpopulations of IPF and normal subjects using molecular typing methods. HLA-DRB1*15 was over-represented in a discovery cohort of 79 Caucasian IPF subjects who had lung transplantations at the University of Pittsburgh (36.7\%) compared to normal reference populations. These findings were prospectively replicated in a validation cohort of 196 additional IPF subjects from four other U.S. medical centers that included both ambulatory patients and lung transplantation recipients. High-resolution typing was used to further define specific HLA-DRB1*15 alleles. DRB1*1501 prevalence in IPF subjects was similar among the 143 ambulatory patients and 132 transplant recipients $(31.5 \%$ and $34.8 \%$, respectively, $\mathrm{p}=0.55)$. The aggregate prevalence of $\mathrm{DRB} 1^{*} 1501$ in IPF patients was significantly greater than among 285 healthy controls (33.1\% vs. $20.0 \%$, respectively, OR $2.0 ; 95 \% \mathrm{Cl} 1.3-2.9, p=0.0004)$. IPF patients with DRB ${ }^{*} 1501(\mathrm{n}=91)$ tended to have decreased diffusing capacities for carbon monoxide $\left(\mathrm{DL}_{\mathrm{CO}}\right)$ compared to the 184 disease subjects who lacked this allele $(37.8 \pm 1.7 \%$ vs. $42.8 \pm 1.4 \%, p=0.036)$.

Conclusions/Significance: DRB1*1501 is more prevalent among IPF patients than normal subjects, and may be associated with greater impairment of gas exchange. These data are novel evidence that immunogenetic processes can play a role in the susceptibility to and/or manifestations of IPF. Findings here of a disease association at the HLA-DR locus have broad pathogenic implications, illustrate a specific chromosomal area for incremental, targeted genomic study, and may identify a distinct clinical phenotype among patients with this enigmatic, morbid lung disease.
\end{abstract}

Citation: Xue J, Gochuico BR, Alawad AS, Feghali-Bostwick CA, Noth I, et al. (2011) The HLA Class II Allele DRB1*1501 Is Over-Represented in Patients with Idiopathic Pulmonary Fibrosis. PLoS ONE 6(2): e14715. doi:10.1371/journal.pone.0014715

Editor: Melanie Koenigshoff, Comprehensive Pneumology Center, Germany

Received July 11, 2010; Accepted January 26, 2011; Published February 23, 2011

This is an open-access article distributed under the terms of the Creative Commons Public Domain declaration which stipulates that, once placed in the public domain, this work may be freely reproduced, distributed, transmitted, modified, built upon, or otherwise used by anyone for any lawful purpose.

Funding: This research was supported in part by the United States National Institutes of Health grants 1RO1HL073241, 1P50 HL084932, 1 P50 HL084948, 5T32 HL007563, and the Intramural Research Program of the National Human Genome Research Institute. The funders had no role in study design, data collection and analysis, decision to publish, or preparation of the manuscript.

Competing Interests: The authors have declared that no competing interests exist.

*E-mail: duncsr@upmc.edu

9 These authors contributed equally to this work.

\section{Introduction}

Idiopathic pulmonary fibrosis (IPF) is a chronic, morbid, fibroproliferative lung disease that manifests with progressive pulmonary restriction and gas exchange abnormalities [1]. The age- and gender-adjusted prevalence of this disease in the U.S. has been recently estimated as 28 to 63 cases per 100,000 persons, and may be increasing [2]. IPF has a dismal prognosis, with a median survival of approximately 3 years after diagnosis, and no medical treatments have proven efficacy $[1,3]$.

Despite extensive investigation, the etiology of IPF remains obscure. Although the pulmonary fibrosis that characterizes this 
disease is often considered to be uniquely dissociated from inflammatory processes [3], studies of patient-derived specimens show that abnormal adaptive immune responses are common in IPF [4-18].

HLA allele frequencies are often aberrantly distributed among patients with immunologic disorders [19-24]. However, HLA characterizations of IPF populations have not been extensively pursued, particularly among disease cohorts that have been delineated by contemporaneous diagnostic criteria [1] and use of definitive, molecular allele typing.

We hypothesized that HLA frequency perturbations may also occur in IPF. Given the apparent prominence of CD4 T-cell responses in IPF [4-9,14-17], we were singularly interested in evaluating the HLA Class II allele frequencies of these patients, since CD4 lymphocytes are predominantly HLA Class IIdependent [25]. Findings of HLA allele biases in IPF could have significant implications for the role of immunogenetic mechanisms in the pathogenesis of this disease.

\section{Methods}

\section{Subjects}

The initial (discovery) IPF cohort consisted of 79 consecutive patients with end-stage pulmonary disease who had molecular HLA allele determinations during evaluations for lung transplantations at the University of Pittsburgh (U. Pgh.), beginning in March 2006.

The subsequent disease validation cohorts were composed of IPF subjects for whom molecular HLA typing results and/or tissue specimens for HLA typing were available from the National Institute of Health (NIH) $(\mathrm{n}=35)$, University of Chicago Medical Center $(\mathrm{n}=32)$, Inova Fairfax Hospital $(\mathrm{n}=20)$, and Stanford University Medical Center $(n=14)$. Subjects from Inova and Stanford were recipients of lung transplantations for end-stage IPF at their respective medical centers. IPF subjects from the NIH and University of Chicago were ambulatory clinic patients. In addition, data were compiled for 19 subjects who had lung transplantations for end-stage IPF at the U. Pgh. subsequent to the initial (discovery) analysis (now current thru October 17, 2010), and 76 ambulatory U. Pgh. IPF Clinic patients.

Diagnoses were prospectively established in the IPF subjects by expert, specialized pulmonary clinicians, blinded to these experimental studies, who analyzed all clinical information, including medical histories and physical exams, pulmonary function tests (PFTs), laboratory studies that included serologic tests for conventional autoimmune syndromes, rheumatologist evaluations, chest radiographs, and computerized tomography (CT) scans that were interpreted by radiologists blinded to other study results. All IPF study subjects fulfilled consensus diagnostic criteria [1]. None had clinical evidence or a past history of connective tissue diseases, drug toxicities, or occupational/environmental exposures associated with interstitial lung disease. Extensive histological evaluations of the diseased pulmonary explants were performed in all cases by blinded, expert lung pathologists. Subjects were excluded if they had histological patterns in their explanted lungs other than usual interstitial pneumonia (UIP) or end-stage fibrotic lung disease [26].

Right-heart catheterizations were routinely performed during assessments for lung transplantations (i.e., among U. Pgh., Inova, and Stanford IPF subjects) by cardiologists who were blinded to these HLA characterizations or knowledge of this study.

Controls consisted of normal Caucasian subjects who had prior molecular HLA typing in the course of other investigations at the U. Pgh. $(\mathrm{n}=196)$ [27] and NIH $(\mathrm{n}=41)$ [28], as well as an additional, prospectively recruited and analyzed U. Pgh. cohort $(\mathrm{n}=48)$. All control subjects were healthy, ambulatory volunteers recruited for study by advertisement and/or solicitation.

Analyses are restricted to Caucasian subjects because $<5 \%$ of lung transplantation recipients in the discovery cohort were members of minority groups, and HLA allele frequencies can vary greatly among racial/ethnic subpopulations [29,30].

Subjects gave written informed consent for these studies that were approved by the respective Institutional Review Boards of all the participating medical centers (e.g., U. Pgh., NIH, U. Chicago, Inova, and Stanford).

\section{HLA Typing}

HLA characterizations of the initial U. Pgh. IPF and normal cohorts were performed using DNA isolated from leukocytes, in sequence specific oligonucleotide probe assays [27]. HLA alleles among the NIH subjects (both IPF and normal) and the prospective U. Pgh. normal cohort $(n=48)$ were evaluated by polymerase chain reaction using sequence specific primers (PCRSSP) (Invitrogen). The presence or absence of DRB1*15 was determined by PCR-SSP among validation IPF subjects at Chicago, Inova, Stanford, and the ambulatory U. Pgh IPF patients. HLA-DRB1* loci typing among those replication cohort specimens that were positive for $\mathrm{DRB} 1 * 15$, as well as high resolution typing of HLA-DRB $1 * 15$ per se were similarly performed by PCR-SSP. Previous study of common specimens confirmed complete concordance of PCR-SSP and oligonucleotide probe assays $(n=40)$.

\section{Statistical Methods}

Allele-disease associations, as well as comparisons of other dichotomous variables, were established by Chi square. Logistic regression analyses were used to generate odds ratios and 95\% confidence intervals. Two-group comparisons of continuous variables were made by two-sample t-test. Factorial ANOVA was used for three or more group comparisons of demographic and physiologic data, with post-hoc comparisons by Bonferroni/ Dunn. Analyses were conducted with StatView v5.0.1 (SAS Institute, Cary, NC). Alpha (p) values $<0.05$ were considered significant. Data are depicted as means $\pm \mathrm{SE}$.

\section{Results}

\section{IPF Subjects}

A total of 275 IPF subjects were studied here (Table 1). The IPF lung transplantation recipients from the U. Pgh., Inova, and Stanford had comparable pulmonary function abnormalities, although U. Pgh. and Chicago subjects tended to be older (Table 1). The extent of pulmonary restriction, ascertained by forced vital capacities as a percentage of predicted values (FVC\%p), was less severe among ambulatory IPF subjects, compared to the transplant recipients $(66 \pm 2 \%$ vs. $53 \pm 1 \%$, respectively, $\mathrm{p}<0.0001)$. Similarly, the percentages of predicted values for single-breath carbon monoxide diffusing capacities $\left(\mathrm{DL}_{\mathrm{CO}} \% \mathrm{p}\right)$, an indicator of intrapulmonary gas exchange, were significantly greater in the ambulatory IPF patients $(48 \pm 2 \%)$ than among those patients who had lung transplantations (33 $\pm 1 \%$ ) $(\mathrm{p}<0.0001)$.

\section{HLA-DRB1*15 Prevalences}

The initial compilation of HLA alleles for the discovery cohort of 79 U. Pgh. transplantation recipients showed the prevalence of DRB $1 * 15$ (calculated as the proportion of subjects who have either one or two copies of this allele) was greater among the IPF patients 
Table 1. IPF Subject Characteristics.

\begin{tabular}{llllllll}
\hline & U. Pgh. Tx. & NIH & U. Chicago & Inova & Stanford & U. Pgh. Amb & Aggregate \\
\hline n & 98 & 35 & 32 & 20 & 14 & 76 & 275 \\
Age (years) & $67 \pm 1$ & $63 \pm 1$ & $69 \pm 1$ & $58 \pm 1$ & $59 \pm 2$ & $70 \pm 1$ & $66 \pm 1$ \\
Males (\%) & 73 & 69 & 90 & 65 & 57 & 74 & 73 \\
Smoking hx (\%) & 66 & 69 & 78 & 55 & 57 & 61 & 65 \\
Status & Tx & Amb & Amb & Tx & Tx & Amb & Both \\
FVC\%p** & $52 \pm 2$ & $74.0 \pm 4$ & $65 \pm 2$ & $55 \pm 4$ & $55 \pm 5$ & $63 \pm 2$ & $60 \pm 1$ \\
DL $_{\text {co\%p }}{ }^{* * *}$ & $32 \pm 1$ & $54 \pm 3$ & $43 \pm 3$ & $36 \pm 4$ & $35 \pm 4$ & $48 \pm 2$ & $41 \pm 1$ \\
DRB1*15 $^{+}(\%)$ & $36(37)$ & $12(34)$ & $10(31)$ & $9(45)$ & $4(29)$ & $24(32)$ & $95(35)$ \\
DRB1*1501 $^{+}(\%)$ & $35(36)$ & $11(31)$ & $10(31)$ & $8(40)$ & $3(21)$ & $24(32)$ & $91(33)$ \\
\hline
\end{tabular}

Tx: transplant recipients; Amb: ambulatory clinic outpatients; hx: history. U.Pgh. Tx includes the 79 subjects of the initial discovery cohort, as well as an additional 19 recipients of lung transplantations for end-stage IPF that have occurred at that institution since the initial discovery compilation. FVC\%p denotes forced vital capacity, as a percentage of predicted normal values; $\mathrm{DL}_{\mathrm{CO}} \% \mathrm{p}$ denotes diffusing capacity for carbon monoxide as a percentage of predicted normal values. Values among $\mathrm{U}$. Pgh. Tx, Inova, and Stanford subjects are based on last determinations immediately prior to their lung transplantations.

$*<0.003$ for U. Pgh. (both Tx. and OP) and/or U. Chicago vs. all others;

$* *<0.003$ for NIH vs. all other groups; U. Pgh. Tx vs. all Amb subpopulations. ${ }^{* * *} \mathrm{p}<0.004$ for NIH vs. all Tx cohorts, U. Pgh. Tx vs. all Amb groups.

doi:10.1371/journal.pone.0014715.t001

than in a normal control population [27] (Table 2). None of the other HLA Class II polymorphisms appeared to be significantly over-represented in these IPF patients (Table 2).

Based on these initial findings, validation cohorts were prospectively compiled, consisting of HLA data from IPF subjects at the NIH and results of DRB $1 * 15$ determinations using blood or tissue specimens from IPF patients at U. Chicago, Inova, Stanford, the IPF outpatient clinic at U. Pgh., as well as an additional volunteer U. Pgh. healthy control cohort. Those IPF and control specimens positive for DRB1*15 were further analyzed to characterize the specific alleles at this locus.

DRB1*15 was present in 64 normal subjects (23\%), and in 95 IPF patients $(35 \%)(\mathrm{p}=0.0015)$. The $\mathrm{DRB} 1 * 1501$ allele accounts for most DRB $1 * 15$ expression in normal Caucasians [29], and similar findings were present in the subjects here. DRB $1 * 1501$ accounted for all but 7 of the DRB1*15 occurrences among the controls and 4 of the IPF patients, and these exceptions were instead attributable to DRB1*1502.

The prevalence of DRB1*1501 within the aggregate IPF replication cohort $(32.1 \%)$ was comparable to that of the original, discovery subjects $(35.4 \%)(p=0.60)$. Likewise, there was no significant difference of $\mathrm{DRB1} * 1501$ prevalence between IPF ambulatory patients $(31.5 \%)$ and IPF transplant recipients $(34.8 \%)$ $(p=0.55)$. The relative distributions of $\mathrm{DRB1}{ }^{*} 1501$ genotype frequencies among the respective control and IPF subpopulations were also similar in both discovery and replication cohorts (Table 3). However, the overall prevalence of this allele among IPF patients was significantly greater than that of the normal controls (Figure 1).

Five IPF patients were known to have first-degree relatives who died with lung disease(s) that could possibly have been IPF. Two of these patients had $\mathrm{DRB} 1 * 1501$. Inasmuch as was known, the remaining IPF cases were due to sporadic disease [1].

Four (4) pulmonary transplantation recipients with pre-operative diagnoses of IPF were excluded from analyses on the basis of lung explant histological evaluation that instead showed single cases each of: pulmonary vasculitic changes suggestive of a clinically unsuspected autoimmune disorder; predominant lymphangitic non-necrotizing granulomas and lymphocytic infiltrates more consistent with hypersensitivity pneumonitis or sarcoidosis; extensive necrotizing granulmatous bronchitis that was highly suggestive of an infectious etiology; and a clinically unsuspected, histoplasma pneumonitis. One of these excluded subjects $(25 \%)$ had DRB1*1501.

Table 2. HLA Class II Allele Prevalence in the Initial IPF Cohort.

\begin{tabular}{|c|c|c|c|}
\hline DQB1* Alleles & IPF Prevalence & $\begin{array}{l}\text { Control } \\
\text { Prevalence }\end{array}$ & p value \\
\hline 02 & 29.1 & 40.6 & 0.07 \\
\hline 03 & 54.4 & 55.7 & 0.70 \\
\hline 04 & 3.8 & 7.8 & 0.23 \\
\hline 05 & 29.1 & 31.8 & 0.67 \\
\hline 06 & 51.9 & 40.1 & 0.08 \\
\hline \multicolumn{4}{|l|}{ DRB1* Alleles } \\
\hline 01 & 17.7 & 21.4 & 0.49 \\
\hline 03 & 20.3 & 21.4 & 0.83 \\
\hline 04 & 29.1 & 28.1 & 0.86 \\
\hline 07 & 21.5 & 26.5 & 0.39 \\
\hline 08 & 3.8 & 8.7 & 0.16 \\
\hline 09 & 1.3 & 1.0 & 0.86 \\
\hline 10 & 2.5 & 1.0 & 0.34 \\
\hline 11 & 16.5 & 20.9 & 0.40 \\
\hline 12 & 2.5 & 3.1 & 0.81 \\
\hline 13 & 24.1 & 25.5 & 0.80 \\
\hline 14 & 5.1 & 3.6 & 0.57 \\
\hline 15 & $36.7^{*}$ & 23.0 & 0.02 \\
\hline 16 & 3.8 & 5.1 & 0.64 \\
\hline
\end{tabular}

HLA allele prevalences (the percentages of subjects with one or more copies of the allele) in the initial U. Pgh. IPF transplant recipient population $(n=79)$ were compared to those of a normal reference population $(n=196)$. DRB $1 * 15$ was the most over-represented of the common HLA Class II alleles among the IPF (bold) relative to the controls. These initial findings prompted further study by recruitments of IPF validation cohorts from four other medical centers and high resolution typing of the DRB1*15 allele (see text).

doi:10.1371/journal.pone.0014715.t002 
Table 3. Genotype distribution of DRB ${ }^{*} 1501$.

\begin{tabular}{|c|c|c|c|c|}
\hline \multirow{2}{*}{$\begin{array}{l}\text { Cohort } \\
\text { Subjects }\end{array}$} & \multicolumn{2}{|l|}{ Discovery } & \multicolumn{2}{|l|}{ Replication } \\
\hline & Control $(n=196)$ & IPF $(n=79)$ & Control $(n=89)$ & IPF $(n=196)$ \\
\hline \multicolumn{5}{|l|}{ Genotype: } \\
\hline DRB1*1501/DRB1*1501 & $1(0.5 \%)$ & $3(3.8 \%)$ & $1(1.1 \%)$ & $3(1.5 \%)$ \\
\hline DRB1*1501/other & 38 (19.4\%) & $25(31.6 \%)$ & $17(19.1 \%)$ & $60(30.6 \%)$ \\
\hline other/other & $157(80.1 \%)$ & $51(64.6 \%)$ & $71(79.8 \%)$ & $133(67.9 \%)$ \\
\hline$p$ value* & 0.0079 & & 0.12 & \\
\hline Allele prevalence (any DRB $1 * 1501$ present) $\mathrm{p}$ value & 0.003 & & 0.035 & \\
\hline
\end{tabular}

Parentheses denote percentages within respective control and IPF subject cohorts. ${ }^{*}$ denotes $\mathrm{p}$ values for the $3 \times 2$ chi-square comparisons of the three genotype distributions among controls vs. IPF within the discovery or replication cohorts, respectively. The $\mathrm{p}$ value of the cumulative $3 \times 2$ chi-square comparison (the three genotype group distributions among all controls vs. all IPF subjects) is 0.0015 .

doi:10.1371/journal.pone.0014715.t003

\section{DRB1*1501 Associations with Clinical Parameters}

There were no apparent associations between the presence $(\mathrm{n}=91)$ or absence $(\mathrm{n}=184)$ of DRB1*1501 among the IPF patients and either age ( $66 \pm 1$ vs. $67 \pm 1$ years) or gender $(75 \%$ vs. $73 \%$ males), for the DRB1*1501 $1^{+}$and $\mathrm{DRB} 1 * 1501^{\text {null }}$ subjects, respectively. Similarly, FVC\%p did not consistently differ between the IPF patients with and without DRB1*1501 (Figure 2A).

However, $\mathrm{DL}_{\mathrm{CO}} \% \mathrm{p}$ values were consistently decreased among the DRB $1 * 1501^{+}$IPF subjects at each study site (Figure $2 \mathrm{~B}$ ). The mean comparative decrement of this clinically relevant physiologic parameter $(31,32)$ was $\sim 12 \%$ among the $\mathrm{DRB} 1 * 1501^{+}$, compared to IPF subjects who did not have this allele. This intergroup difference seemed unlikely to be a cryptic result of smoking per se, as near equal proportions of IPF subjects with and without DRB1*1501 had smoking histories (67\% and 64\%, respectively) and cumulative smoke exposures (23 3 and $21 \pm 2$ pack-years, respectively). $\mathrm{DL}_{\mathrm{CO}}$ abnormalities can also be attributable to

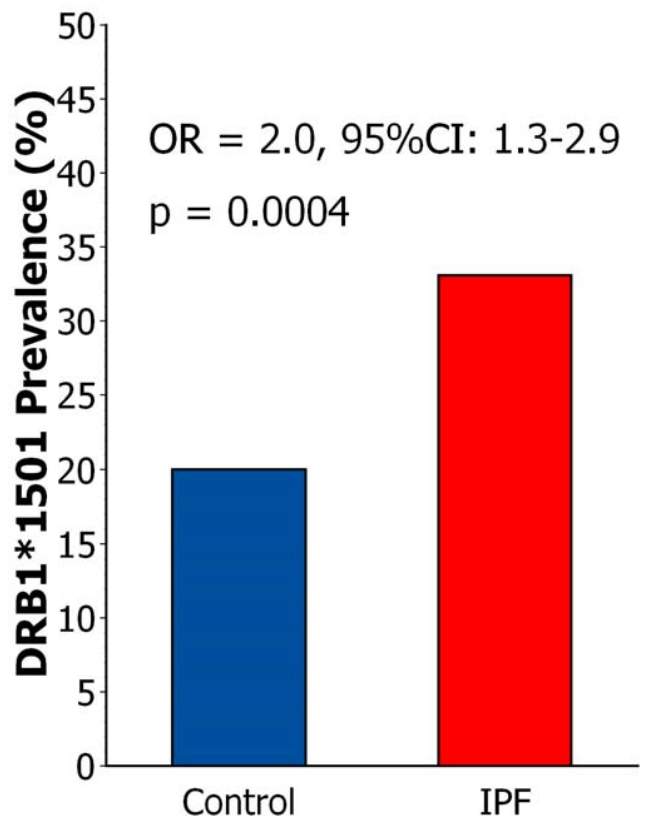

Figure 1. DRB1*1501 prevalence in IPF and controls. DRB1*1501 was significantly over-represented in the cumulative IPF population $(n=275)$ compared to healthy controls $(n=285)$.

doi:10.1371/journal.pone.0014715.g001 secondary effects of pulmonary artery hemodynamics [33]. Nonetheless, we found no intergroup differences of pulmonary artery pressures based on the presence or absence of $\mathrm{DRB} 1 * 1501$ among those IPF patients who had right heart catheterizations (i.e., the transplantation recipients from the U. Pgh., Inova, and Stanford), with respect to systolic $(41 \pm 2$ vs. $43 \pm 2 \mathrm{~mm} \mathrm{Hg}$, respectively), diastolic (14 \pm 1 vs. $15 \pm 1 \mathrm{~mm} \mathrm{Hg}$, respectively) or mean pressures (25 \pm 1 vs. $26 \pm 1 \mathrm{~mm} \mathrm{Hg}$, respectively).

\section{Discussion}

These findings show the HLA Class II allele DRB1*1501 is over-represented among Caucasian IPF subjects with highly variable disease severities at multiple U.S. medical centers (Figure 1). The presence of DRB1*1501 in IPF patients also seems associated with greater magnitudes of gas exchange impairment (Figure 2B). The present findings implicate immunoregulatory elements within the HLA Class II complex in the pathogenesis of IPF. These data are also consistent with the hypothesis that the development of IPF likely involves interactions between environmental agent(s) and genetic factors [34,35], a disease paradigm also common to many other disorders, notably including those caused by aberrant immune processes [19-24].

Two distinct mechanisms may account for the frequently observed associations between unique HLA polymorphisms and various disease syndromes [19-24]. First, HLA molecules are requisite effectors for presentations of peptide antigens to the $\mathrm{T}$ cells that initiate adaptive immune responses, but each distinct HLA allele has a restricted peptide binding motif [25]. Hence, HLA haplotype inheritance determines the finite repertoire of antigens that can evoke T-cell responses in an individual. Although critical for host defense, these adaptive immune responses may be deleterious if, as an example, the antigen is a self-protein (autoantigen), or one that evokes a cross-response to a self-protein $[25,36,37]$. In contrast, individuals lacking these specific, "permissive" HLA alleles do not present those particular antigens, and do not initiate the deleterious response(s).

Alternatively, over-representation of a specific HLA molecule(s) in a disease cohort may be essentially unrelated to the unique antigen presentations of that HLA per se, but is instead a genetic "marker" denoting the presence of a pathogenic immunomodulatory gene(s) that is(are) in strong linkage disequilibrium (LD) with that HLA allele [21,38].

The human major histocompatibility (MHC) complex on chromosome $6 \mathrm{p} 21.31$ is characterized by the presence of numerous, extraordinarily polymorphic HLA alleles, and many other proxi- 

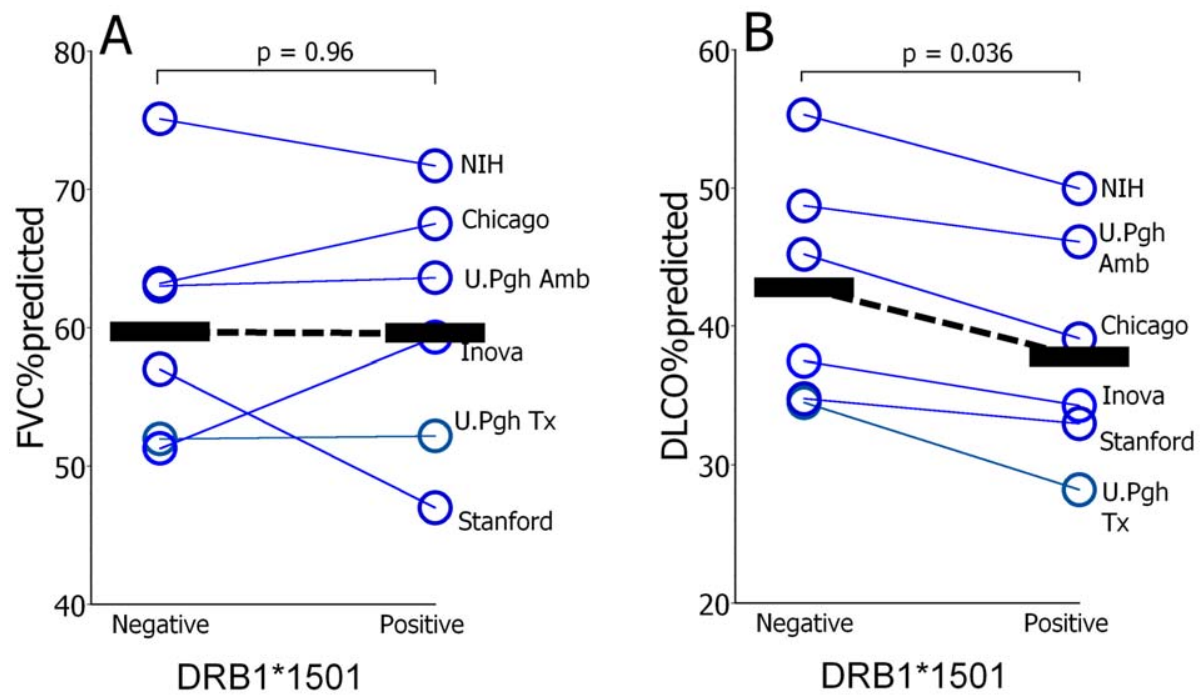

Figure 2. Associations of DRB1*1501 and lung function. A.) Forced vital capacities, as percentages of predicted normal values (FVC\%predicted), did not show a consistent association with the presence or absence of DRB1*1501 among the IPF subjects. Aggregate mean values are denoted by horizontal bars linked by dashed lines. B.) Diffusing capacities, as percentages of predicted normal values (DL $L_{c o} \%$ predicted), were decreased among those IPF subjects with DRB $1 * 1501$ vs. those patients who did not have this allele, at each participating center. These differences were significant in comparisons of the cumulative (aggregate) IPF populations, despite considerable overall differences of $D L_{c o} \%$ predicted values (e.g., "noise") between the various participating medical centers (see also Table 1).

doi:10.1371/journal.pone.0014715.g002

mate immunoregulatory genes that are often in very strong LD [19$21,26,38]$. DRB $1 * 1501$ is in nearly complete LD with DQA $1 * 0102$ and DQB1*0602 among Caucasians, and is the single most frequent HLA Class II haplotype in this racial group [29]. Overrepresentation of the DRB1*1501 HLA allele (and/or the DRB1*1501-DQA1*0102-DQB1*0602 haplotype) is also one of the most frequently reported genetic findings of patients with diverse immunologic diseases, including Goodpasture's syndrome, multiple sclerosis, systemic lupus erythematosus (SLE), and sarcoidosis [1924]. Because of the very strong LD within this haplotype, it is difficult to precisely identify the particular disease-associated HLA allele among them, or distinguish the contributions of these HLA from other interspersed immunoregulatory elements, in lieu of focused, high-resolution genomic studies [21,38,39].

Almost all previous HLA characterizations of IPF patients date from before the development of precise and definitive molecular methodologies that distinguish these alleles [40-45] (Table 4). Moreover, only two of these early serologic-based determinations examined even a very restricted repertoire of the many, sincediscovered, Class II alleles [44,45]. Furthermore, diagnostic criteria for IPF have evolved considerably during the intervening years [1], raising potential concerns about the case definitions of the earlier study populations. The numbers of subjects among those investigations were also usually quite small (Table 4), severely limiting their power to detect intergroup differences. Despite these potential limitations, however, several of those earlier investigations indicated HLA allele frequency perturbations may be present in IPF [40,43-45], although this finding was not invariable $[41,42]$. In particular, one of these earlier studies indicated that DR2, a serologic correlate of HLA-DRB1*15 and, generally much less frequently, HLA-DRB $1 * 16$ gene products, appeared to be over-represented in IPF subjects (45), a finding which may be congruent with the current results.

To our knowledge, contemporary analogous analyses using molecular techniques and current IPF case definitions are limited to a single cohort study of Mexican patients that reported various HLA alleles, including DRB $1 * 01, \mathrm{DRB} 1 * 04$, and DRB $1 * 14$, were over-represented in IPF [46]. We did not see abnormal frequencies of those particular DRB $1 *$ alleles $(\mathrm{DRB} 1 * 01$, *04, or *14) in our IPF population (Table 2). Conversely, the frequencies of DRB1*15 alleles in both the IPF and normal control populations of that previous study [46] were several-fold less than that measured here, and were also much less than frequencies reported within other large Caucasian control populations $[22,24,29,30]$. The seeming discrepancy between that previous report and the present findings may be attributable to the often considerable variability of HLA allele frequencies among different races and ethnicities [19-21,29].

The present study has several unique aspects. To our knowledge, the number of subjects with this uncommon disease that were analyzed here is unprecedented among analogous investigations (Table 4). The findings of DRB1*15 over-representation in the discovery U. Pgh. cohort with end-stage lung disease was also uniquely validated in prospective replications of subjects from four other participating medical centers, and included IPF patients with highly variable disease severities. The present study is also unusual in that, in addition to adherence with current diagnostic criteria [1], extensive histological evaluations of entire lung explants were available for the many disease subjects here who had lung transplantations, thereby further ensuring accuracy of these case definitions.

Other findings here that $\mathrm{DL}_{\mathrm{CO}}$ tended to be comparatively less among those IPF patients with DRB1*1501 (Figure 2B), particularly in the absence of confounding by intergroup differences of smoking exposures or pulmonary artery pressures, could perhaps imply that the role ultimately played by this immunogenetic factor(s) may have a singularly virulent effect with respect to the lung injury mechanisms that result in gas exchange impairment. Decrements of $\mathrm{DL}_{\mathrm{CO}}$ are widely used indices of disease activity and prognosis [31], and may be useful ancillary indications for lung transplantation among IPF patients [32].

Although IPF is not widely considered to be an immunologic disorder, recent studies of patient-derived clinical specimens reveal the presence of abnormal adaptive immune responses among 
Table 4. Published studies of HLA allele frequencies in IPF.

\begin{tabular}{|c|c|c|c|c|c|c|}
\hline Loci & \#Alleles tested & $\begin{array}{l}\text { Molecular } \\
\text { diagnoses }\end{array}$ & IPF (n) & Abnormal in IPF? & year & ref \\
\hline$?$ & ? & No & $20^{*}$ & Yes HLA12 & 1976 & 40 \\
\hline$-A,-B$ & 24 & No & 32 & no & 1977 & 41 \\
\hline$-A,-B$ & 35 & No & 33 & no & 1978 & 42 \\
\hline$-A,-B,-C$ & 36 & No & 50 & Yes: B8 & 1978 & 43 \\
\hline $\begin{array}{l}-A,-B,-C, \\
-D w\end{array}$ & $\begin{array}{l}32 \\
4\end{array}$ & No & $38^{*}$ & Yes: B15 and Dw6 & 1979 & 44 \\
\hline$-A,-B,-C,-D R$ & 65 (total in all loci) & No & 20 & Yes: DR2 & 1983 & 45 \\
\hline$-A,-B,-D R,-D Q$ & 45 multiple & Yes & 75 & $\begin{array}{l}\text { Yes, multiple Class I and Class II alleles and } \\
\text { haplotypes (but not DRB } 1^{*} 15 \text { ) }\end{array}$ & 2005 & 46 \\
\hline
\end{tabular}

*included patients with other autoimmune syndromes. Approximately fifty (50) HLA-A, 85 HLA-B, 45 HLA-C, 44 HLA-DR, and 16 HLA-DQ distinct polymorphisms (alleles and suballeles) are currently known to be expressed in Caucasian populations [29].

doi:10.1371/journal.pone.0014715.t004

those afflicted by this disease. The majority of IPF patients have IgG autoantibodies against various autoantigens that are typically distinct from those described in classical autoimmune syndromes (e.g., SLE, scleroderma, etc.) [5-8,9,14], and the presence of particular autoantibody responses in individual IPF patients have been associated with clinical manifestations of their lung disease $[4,5,9]$. In addition, T-cells among IPF patients show increased extents of prior activation, enhanced production of various inflammatory and/or pro-fibrotic mediators (e.g., TGF- $\beta 1$ ), and impaired regulatory $\left(T_{\text {reg }}\right)$ function $[10-14,17]$. Moreover, firstdegree relatives of patients with familial IPF show intrapulmonary infiltrations of activated CD4 T-cells many years prior to the development of clinically-evident lung abnormalities [15]. Activated, pro-inflammatory dendritic cells with augmented ability to present antigens to $\mathrm{T}$-cells also accumulate in the pulmonary parenchyma of IPF patients [18]. CD4 T-cell oligoclonal proliferation is extensive in the lungs and periphery of IPF patients, a highly specific finding of repetitive lymphocyte stimulation by a restricted set of conventional peptide antigens $[13,14]$. Abnormal proportions of phenotypically and functionally distinct $\mathrm{CD} 4^{+} \mathrm{CD} 28^{\text {null }} \mathrm{T}$-cells, the daughter progeny of repetitive antigen-driven lymphocyte proliferations, and a frequent and specific finding of chronic immunologic diseases [47,48], are also present in the circulation (and lungs) of IPF patients singularly destined for poor outcomes [16]. Moreover, intrapulmonary peptide antigen $(\mathrm{s})$ isolated from diseased IPF lungs uniquely drive proliferations of autologous CD4 T-cells [14].

Considerable efforts have been directed towards discovery of the genetic determinants for IPF $[34,35]$. The present data show that the HLA Class II complex, a unique chromosomal region with extreme polymorphism, unusually strong, nonrandom LD, and a high density of diverse immunoregulatory genes [21,38,39], include one or more loci involved in IPF pathogenesis. These findings justify further, specific characterizations of targeted HLA region genes and polymorphisms among IPF cohorts, and correlative clinical and immunologic functional studies, in order to ultimately discern the processes that contribute to development and/or progression of this devastating, intractable disease.

\section{Author Contributions}

Conceived and designed the experiments: JX IOR VGV YZ SD. Performed the experiments: JX BRG ASA CFB SDN GR IOR SD IO CF MAS SSJ AZ PAM JMP FCS YZ SD. Analyzed the data: BRG ASA CFB IN SDN GR IOR SD IO CF KC MAS SSJ AZ PAM JMP VGV
FCS YZ SD. Contributed reagents/materials/analysis tools: BRG ASA CFB IN SDN GR IOR SD IO CF KC MAS SSJ AZ PAM JMP VGV KFG NK FCS YZ SD. Wrote the paper: JX KC PAM KFG SD. Proofed and/or edited paper: JX BG AS CF-B SDN GR SD IO CF KC MAS SSJ AZ JMP VGV KFG NK YZ FCS Performed the brunt of the specimen processing, DNA extraction, HLA assays and validations of same. Assisted with data interpretations and analyses: JX. Identified NIH normal and IPF subjects, recruited them, obtained and processed specimens, and compiled and analyzed relevant clinical and HLA data for this validation cohort: BG. Assisted with specimen collation, DNA purification from same, and with HLA assays: AS. Assisted with HLA typing assays, contributed to experimental design features: CF-B. Identified Inova IPF subjects, enrolled them, obtained and processed specimens, and compiled and analyzed relevant clinical data for a validation cohort: SDN. Identified Stanford IPF subjects, enrolled them, obtained and processed specimens, and compiled and analyzed relevant clinical data for a validation cohort: GR. Performed blinded interpreations of surgical pathology specimens that validated their diagnoses of IPF: SD. In conjunction with Dr. Fuhrman, interpreted and validated chest CT scans of IPF patients: IO. Provided blinded radiographic validations of IPF chest CT scans: CF. Provided information critical for study design, assisted with and validated data analyses: KC. In conjunction with Dr. Nathan, identified Inova subjects, enrolled them, obtained and processed specimens, and compiled and analyzed relevant clinical data for a validation cohort from Inova: MAS. In conjunction with Dr. Rosen, identified Stanford subjects, enrolled them, obtained and processed specimens, and compiled and analyzed relevant clinical data for a validation cohort from Stanford: SSJ. Obtained and processed specimens of the U. Pgh. transplant population, performed HLA analyses of same. Provided additional specimens for high resolution typing as well as technical advice: AZ. Identified control subjects, enrolled them, obtained and processed specimens, performed HLA analyses, and compiled and analyzed relevant clinical data for the initial discovery control cohort. Assisted with data interpretations and manuscript writing: PAM. Identified transplant recipient subjects, enrolled them, obtained and processed specimens, and compiled and analyzed relevant clinical data for the initial IPF discovery cohort. Facilitated or procured specimen acquisitions from same: JMP. Analyzed data, assisted with study design and identifications of collaborators and validation cohorts. Assisted with data analyses. Procured additional data: VGV. Identified ambulatory IPF subjects, recruited them for these studies, obtained and processed specimens, and compiled and analyzed relevant clinical data for this validation cohort: KFG NK. Provided technical assistance and advice. Helped with DNA isolation and analyses: YZ. Identified normal volunteer subjects, enrolled them, obtained and processed specimens, and compiled and analyzed relevant clinical data for a validation control cohort: FCS. Conceived and designed experiments, coordinated specimen acquistion, processing and analyses, collated clinical information provided by colleagues, analyzed the data in conjunction with co-authors, provided financial support of the studies, and wrote the paper: SRD. 


\section{References}

1. American Thoracic Society (2000) Idiopathic pulmonary fibrosis: diagnosis and treatment. International consensus statement. American Thoracic Society (ATS), and the European Respiratory Society (ERS). Am J Respir Crit Care Med 161: 646-664.

2. Perez EF, Daniels CE, Schroeder DR, St. Sauver J, Harman TE, et al. (2010) Incidence, prevalence, and clinical course of idiopathic pulmonary fibrosis: a population-based study. Chest 137: 129-137.

3. Selman M, Thannickal VJ, Pardo A, Zisman DA, Martinez FJ, et al. (2004) Idiopathic pulmonary fibrosis: pathogenesis and therapeutic approaches. Drugs 64: 406-430.

4. Kurosu K, Takiguchi Y, Okada O, Yumoto N, Sakao S, et al. (2008) Identification of annexin 1 as a novel autoantigen in acute exacerbation of idiopathic pulmonary fibrosis. J Immunol 181: 756-767.

5. Ogushi F, Tani K, Endo T, Tada H, Kawano T, et al. (2001) Autoantibodies to IL- $1 \alpha$ in sera from rapidly progressive idiopathic pulmonary fibrosis. J Med Invest 48: 181-9.

6. Magro CM, Waldman WJ, Knight DA, Allen JN, Nadasdy T, et al. (2006) Idiopathic pulmonary fibrosis related to endothelial injury and antiendothelial cell antibodies. Hum. Immunol 67: 284-297.

7. Takahashi T, Wada I, Ohtsuka Y, Munakata M, Homm Y, et al. (2007) Autoantibody to alanyl-tRNA synthetase in patients with idiopathic pulmonary fibrosis. Respirology 12: 642-653.

8. Dobashi N, Fujita J, Murota M, Ohtsuki Y, Yamadori I, et al. (2000) Elevation of anti-cytokeratin 18 antibody and circulating cytokeratin 18: anti-cytokeratin 18 antibody immune complexes in sera of patients with idiopathic pulmonary fibrosis. Lung 178: 171-179.

9. Taillé C, Grootenboer-Mignot S, Boursier C, Michel L, Debray MP, et al. (2010) Identification of periplakin as a new target for autoreactivity in idiopathic pulmonary fibrosis. Am J Respir Crit Care Med. Oct 8. [Epub ahead of print].

10. Papiris SA, Kollintza A, Karatza M, Manali ED, Sotiropoulou C, et al. (2007) CD8+ $\mathrm{T}$ lymphocytes in broncholaveolar lavage in idiopathic pulmonary fibrosis. J Inflamm (Lond) 4: 14-18.

11. Marchal-Somme J, Uzunhan Y, Marchand-Adam S, Valeyre D, Soumelis V, et al. (2006) Cutting edge: non-proliferating mature immune cells form a novel type of organizing lymphoid structure in idiopathic pulmonary fibrosis. J Immunol 176: 5735-5739.

12. Homolka J, Ziegenhagen MW, Gaede KI, Entzian P, Zissel G, et al. (2007) Systemic immune cell activation in a subgroup of patients with idiopathic pulmonary fibrosis. Respiration 70: 262-269

13. Shimizudani A, Murata H, Keino H, Kojo S, Nakamura H, et al. (2002) Conserved CDR 3 region of $\mathrm{T}$ cell receptor $\mathrm{BV}$ gene in lymphocytes from bronchoalveolar lavage fluid of patients with idiopathic pulmonary fibrosis. Clin Exp Immunol 129: 140-149.

14. Feghali-Bostwick CA, Tsai CG, Valentine VG, Kantrow S, Stoner MW, et al. (2007) Cellular and humoral autoreactivity in idiopathic pulmonary fibrosis. J Immunol 179: 2592-9.

15. Rosas IO, Ren P, Avila NA, Chow CK, Franks TJ, et al. (2007) Early interstitial lung disease in familial pulmonary fibrosis. Am J Resp Crit Care Med 176: 698-705.

16. Gilani SR, Vuga LJ, Lindell KO, Gibson KF, Xue J, et al. (2010) CD28 downregulation on circulating CD4 T-cells is associated with poor prognoses of patients with idiopathic pulmonary fibrosis. Plos One 2010; 5: e8959.

17. Kotslanidis I, Nakou E, Bouchliou I, Tzouvelekis A, Spanoudakis E, et al. (2009) Global impairment of $\mathrm{CD} 4^{+} \mathrm{CD} 25^{+} \mathrm{FoxP} 3^{+}$regulatory $\mathrm{T}$ cells in idiopathic pulmonary fibrosis. Am J Resp Crit Care Med 179: 1121-1130.

18. Marchal-Somme J, Uzunhan Y, Marchand-Adam S, Kambouchner M, Valeyre D, et al. (2007) Dendritic cells accumulate in human fibrotic interstitial lung disease. Am J Respir Crit Care Med 176: 1007-1014.

19. Hall FC, Bowness P (1996) HLA and disease: From molecular function to disease association? In HLA and MHC: Genes, molecules, and function. Browning MJ, McMichael AJ, eds. Oxford: BIOS Scientific Publishers Ltd. pp 353-381.

20. Warrens A, Lechler R (1999) HLA in health and disease. San Diego: Academic Press.

21. de Bakker PI, McVean G, Sabeti PC, Miretti MM, Green T, et al. (2006) A high-resolution HLA and SNP haplotype map for disease association studies in the extended human MHC. Nature Genetics 38: 1166-1172.

22. Schmidt H, Williamson D, Ashley-Koch A (2007) HLA-DR15 haplotype and multiple sclerosis: a huge review. Am J Epidemiol 165: 1097-1109.
23. Takeuchi F, Nakano K, Nabeta H, Hong GH, Kawasugi K, et al. (2005) Genetic contribution of the tumor necrosis factor (TNF) B $+252 * 2 / 2$ genotype, but not the TNFa,b microsatellite alleles, to system lupus erythematosis in Japanese patients. Int J Immunognet 32: 173-178.

24. Voorter CEM, Drent M, van den Berg-Loonen EM (2005) Severe pulmonary sarcoidosis is strongly associated with the haplotype HLA-DQB $1 * 0602$ DRB1*1501. Hum Immunol 66: 826-835.

25. Rudolph MG, Stanfield RL, Wilson IA (2006) How TCRs bind MHCs, peptides, and coreceptors. Annu Rev Immunol 24: 419-466.

26. Katzenstein A-L, Myers JL (1988) Idiopathic pulmonary fibrosis. Clinical relevance of pathologic classification. Am J Respir Crit Care Med 157: 1301-1315.

27. Falkner D, Wilson J, Fertig N, Clawson K, Medsger TA, et al. (2000) Studies of HLA-DR and DQ alleles in SSc patients with autoantibodies to RNA polymerases and U3-RNP (fibrillarin). J Rheum 27: 1196-1201.

28. Ren P, Rosas IO, MacDonald SD, Wu HP, Billings EM, et al. (2007) Impairment of alveolar macrophage transcription in idiopathic pulmonary fibrosis. Am J Respir Crit Care Med 175: 1151-7.

29. National Bone Marrow Donor Program. Haplotype Frequencies. https:// bioinformatics.nmdp.org. Accessed 2010 April 10.

30. Klitz W, Maiers W, Spellman S, Baxter-Lowe LA, Schmeckpeper B, et al. (2003) New HLA haplotype frequency reference standards: High-resolution and large sample typing of HLA-DR-DQ haplotypes in a sample of European Americans. Tissue Antigen 62: 296-307.

31. Collard HR, King TE, Bartelson BB, Vourlekis JS, Schwartz MI, et al. (2004) Changes in clinical and physiologic variable predict survival in idiopathic pulmonary fibrosis. Am J Respir Crit Care Med 169: 538-542.

32. Kreider M, Kotloff RM (2009) Selection of candidates for lung transplantation. Proc Am Thorac Soc 6: 20-27.

33. Lettieri CJ, Nathan SD, Barnett SD, Ahmad S, Shorr AF (2006) Prevalence and outcomes of pulmonary arterial hypertension in advanced idiopathic pulmonary fibrosis. Chest 129: 746-752.

34. Grutters JC, du Bois RM (2005) Genetics of fibrosing lung diseases. Eur Resp J 25: 915-927.

35. Lawson WE, Loyd JE (2006) The genetic approach in pulmonary fibrosis. Can it provide clues to this complex disease? Proc Am Thorac Soc 3: 345-349.

36. Oldstone MB (2005) Molecular mimicry, microbial infection and autoimmune disease: evolution of the concept. Curr Top Microbiol Immunol 296: 1-17.

37. Vanderlugt CL, Miller SD (2002) Epitope spreading in immune mediated diseases: implications for immunotherapy. Nat Rev Immunol 2: 85-94.

38. Stewart CA, Horton R, Allcock RJN, Ashurst JL, Atrazhev AM, et al. (2004) Complete MHC haplotype sequencing for common disease gene mapping. Genome Res 14: 1176-1187.

39. Traherne JA, Horton R, Roberts AN, Miretti MM, Hurles ME, et al. (2006) Genetic analysis of completely sequenced disease-associated MHC haplotypes identifies shuffling of segments in recent human history. PLoS Genetics 2: 0081.

40. Evans C (1976) HLA antigens in diffuse fibrosing alveolitis. Thorax 31: 483-5.

41. Strimlan CV, Taswell HF, DeRemee RA, Kueppers F (1977) HLA antigens and fibrosing alveolitis. Am Rev Resp Dis. pp 1120-1.

42. Fulmer JD, Sposovska MS, von Gal ER, Crystal RG, Mittal KK (1978) Distribution of HLA antigens in idiopathic pulmonary fibrosis. Am Rev Resp Dis 118: 141-47.

43. Turton CWG, Morris LM, Lawler SD, Turner-Warwick M (1978) HLA in cryptogenic fibrosing alveolitis. Lancet 1: (8062): 507-8.

44. Varpela E, Tiilkainen A, Varpela M, Tukiainen P (1979) High prevalences of HLA-B15 and HLA-Dw6 in patients with cryptogenic fibrosing alveolitis. Tissue Antigens 14: 68-71.

45. Libby DM, Gibofsky A, Fotino M, Waters SJ, Smith JP (1983) Immunogenetic and clinical findings in idiopathic pulmonary fibrosis. Am Rev Resp Dis 127: 618-22.

46. Falfan-Valenci R, Camarena A, Juarez A, Becerril C, Montano M, et al. (2005) Major histocompatibility complex and alveolar epithelial apoptosis in idiopathic pulmonary fibrosis. Hum Genet 118: 235-244.

47. Vallejo AN, Weyand CM, Goronzy JJ (2004) T-cell senescence: a culprit of immune abnormalities in chronic inflammation and persistent infection. Trends Mol Med 10: 119-124.

48. Studer SM, George MP, Zhu X, Song Y, Valentine VG, et al. (2008) CD28 downregulation on CD4 T-Cells is a marker for graft dysfunction in lung transplant recipients. Am J Resp Critical Care Med 178: 765-773. 